\title{
Biofilms of streptococci and veillonellae: interactions in vitro and in the oral cavity.
}

\author{
Robert J. Palmer Jr., Paul G. Egland, and Paul E. Kolenbrander
}

Oral Infection and Immunity Branch, National Institute of Dental and Craniofacial Research, National Institutes of Health, Bethesda, MD 20892

Streptococci make up 60-90\% of the cultivable bacterial biomass on human supragingival tooth surfaces. Veillonellae make up about $5 \%$ of the biomass. Streptococci ferment carbohydrates to a mixture of short-chain organic acids of which lactic acid is a major component. Veilllonellae cannot use carbohydrates as a carbon source. Instead, they metabolize organic acids, e.g., by fermenting lactic acid to propionate, hydrogen, and $\mathrm{CO}_{2}$. It has been hypothesized that, within dental plaque biofilms, veillonellae depend upon streptococci. Indeed, more veillonellae were recovered from the mouths of gnotobiotic rats that received an oral co-inoculum of streptococci and veillonellae than from mouths of rats that received solely veillonellae [1]. In addition, the rats that were co-inoculated had lower caries levels than those without veillonellae, which suggests that veillonellae reduce lactic acid concentration at tooth surfaces and thereby prevent enamel dissolution [2].

We examine streptococci-veillonellae interactions in biofilms using a perfusion chamber. After a 15min attachment period, $25 \%$ human saliva is pumped through the chamber to remove unattached bacteria and to provide the sole nutrient source for the growing biofilm. Biofilm biomass is quantified using laser confocal microscopy and 3D image analysis, and lactic acid concentration in the effluent is measured fluorometrically. These experiments demonstrate that veillonellae do not grow as a monoculture biofilm, but that streptococci do. In a monoculture biofilm, Streptococcus oralis strain 34 produces lactic acid. When this strain and veillonellae are grown as a co-culture biofilm, the streptococci and veillonellae both grow, but lactic acid is undetectable. S. oralis strain J22 does not produce lactic acid in monoculture, but still supports growth of veillonellae in a co-culture biofilm. These observations suggest that the small amount of carbohydrate present in saliva is sufficient for streptococci to produce the lactic acid required for growth of veillonellae, and that lactic acid is not the only streptococcal metabolite that supports veillonellae growth.

S. gordonii strain V288 synthesizes a starch-like intracellular polysaccharide. It also possesses an intracellular amylase activity that is regulated by sugar intermediates and that can hydrolyze starch. When V288 is grown on agar plates that reveal starch hydrolysis, little reactivity is seen. However, when grown as a mixed culture with Veillonella strain PK1910, a reaction does occur. These data indicated that PK1910 causes up-regulation of V288 amylase activity. A GFP reporter system was designed to detect amylase gene activation. Flow cytometric analysis of suspension cultures reveal that V288 makes little GFP. However, when grown in suspension together with PK1910, V288 makes GFP. Of particular interest is that GFP is also produced when the two bacteria are grown as individual suspension cultures separated by a dialysis membrane. Furthermore, when V288 and PK1910 are grown together as a biofilm in the previously described saliva-fed perfusion chamber, laser confocal microscopy demonstrates that GFP is produced only by V288 cells that are in direct contact with PK1910 cells (Fig. 1). These results indicate that veillonellae communicate with streptococci to increase amylase activity, the communication involves a diffusible signal, and that close proximity between the bacteria is required to increase amylase activity within biofilms grown under flowing conditions. Dilution of the signal by flowing saliva results in a signal concentration too low to trigger amylase activity unless the two cell types are in intimate juxtaposition to one another [3].

We also study streptococci-veillonellae interactions in natural human plaque. A small chip of tooth enamel is worn in a stent on a volunteer's lower jaw. After 4 or $8 \mathrm{hrs}$, the chip is removed, the 
biofilm is stained, and the architecture of the biofilm is examined using laser confocal microscopy. Early plaque contains cells reactive solely with the broadly reactive anti-viellonella antibody anti-R1, but not with the more selective anti-veillonella antibody anti-1910. Later biofilms contain cells reactive exclusively with anti-1910, but also contain cells co-reactive with anti-R1 and anti-1910. Veillonellae are intimately associated with streptococci in the plaque. These data show that veillonellae and streptococci form mixed-species communities in situ within human oral biofilms (Fig. 2 , left panel), and that the population of veillonellae evolves in diversity within the short period of eight hours (Fig. 2, right panel) [4]: a period that coincides with typical daily oral hygiene cycles.

This research was supported by the Intramural Research Program of the National Institute for Dental and Craniofacial Research at the National Institutes of Health.

[1] B. C. McBride \& J. S. van der Hoeven, Infect. Immun. 33 (1981) 467.

[2] J. S. van der Hoeven et al., Caries Res. 12 (1978) 142.

[3] P. G. Egland et al., Proc. Natl. Acad. Sci. U. S. A. 101 (2004) 16917.

[4] R. J. Palmer, Jr. et al., J. Bacteriol. 188 (2006) 4117.
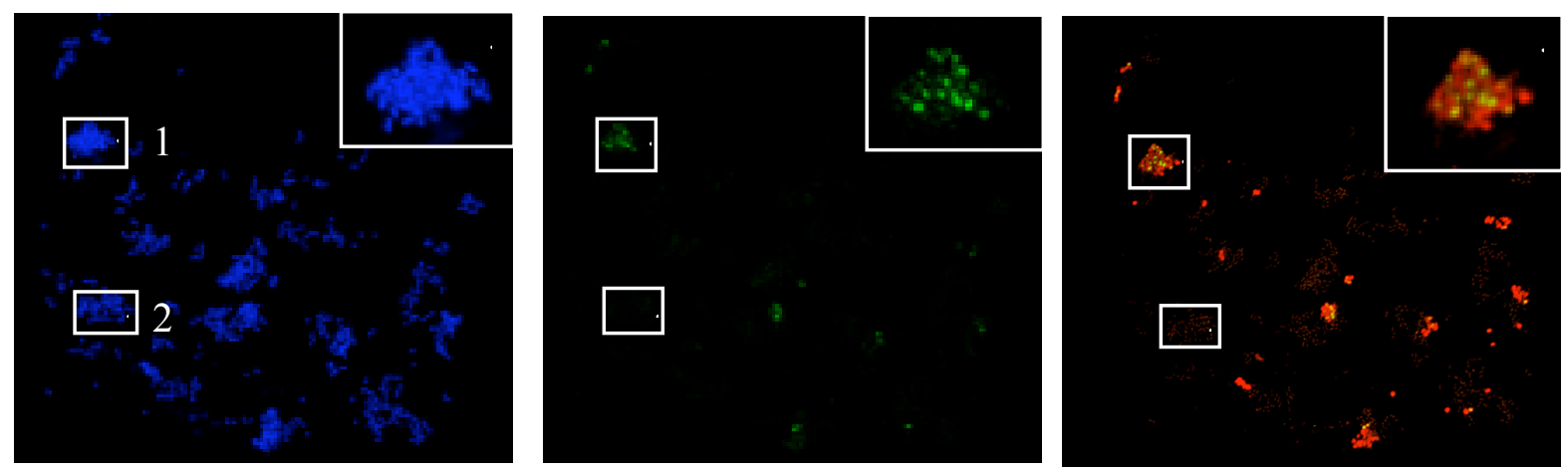

Figure 1. All images are the same field-of-view. Left: all cells stained with Syto 19. Middle: GFPproducing streptococci. Right: Overlay of anti-veillonella stained cells (red) and GFP-producing streptococci. Box 1 and inset at upper right: a mixed community in which streptococci produce GFP also contains veillonellae. Box 2: Streptococcal cells not in contact with veillonellae produce no GFP.
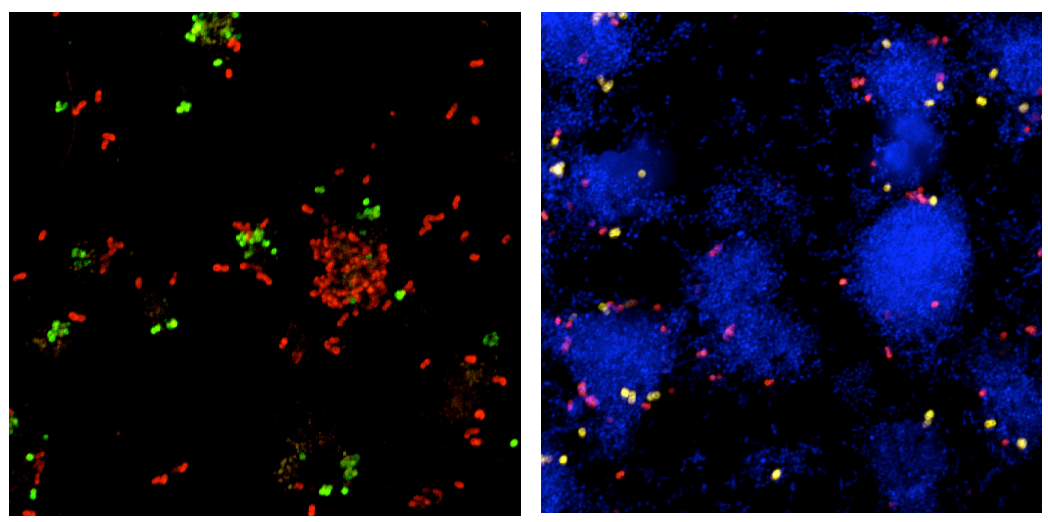

Figure 2. Left: 18-hr plaque stained with anti-streptococcus (red) and anti-1910 (green). Right: 18-hr plaque stained with DAPI (blue), anti-R1 (green) and anti1910 (red). Note presence of red cells (reactive solely with anti-R1), presence of yellow cells (coreactive with both antibodies), and absence of green cells (reactive solely with anti-1910). 